\title{
Perspectives on Type III Statistical Errors: Exaggerating the Effects of Placebo in Neurofeedback
}

\author{
Mark Trullinger ${ }^{1,2^{*}}$, Allen Novian ${ }^{3,4}$, Lori Russell-Chapin ${ }^{5,6}$, and Deepti Pradhan ${ }^{1,2}$ \\ ${ }^{1}$ The Chicago School of Professional Psychology, Washington, District of Columbia, USA \\ ${ }^{2}$ NeuroThrive, LLC, Lutherville, Maryland, USA \\ ${ }^{3}$ St. Mary's University, San Antonio, Texas, USA \\ ${ }^{4}$ integrative Counseling \& Neurofeedback Solutions (iCNS), San Antonio, Texas, USA \\ ${ }^{5}$ Bradley University, Peoria, Illinois, USA \\ ${ }^{6}$ Neurotherapy Institute of Central Illinois, Peoria, Illinois, USA
}

\section{Abstract}

Evaluating the efficacy of electroencephalography neurofeedback (EEG-nf) for the treatment of attentiondeficit/hyperactivity disorder (ADHD) has been a topic of vigorous debate over the past few years. However, many of the articles state a lack of efficacy and insist on placebo as the explanation for any positive effects found in the EEG-nf treatment group. Several critical flaws in this analysis are discussed including the existence of non-inert shams, the false no-effect, and placebo as an ad hoc explanation. These flaws lead to Type III statistical errors, which are often repeated in other articles. It is recommended that journals, books, and media articles publishing new research and reviews on the efficacy of EEG-nf be vigilant for these errors in order to improve the quality of the EEG-nf body of research. Requiring researchers and authors reviewing the literature to verify assumptions of non-inert shams, ensure the use of best practices in the EEG-nf treatment groups, and clearly identify ad hoc conclusions can avoid these Type III errors.
\end{abstract}

Keywords: EEG-nf; sham; false no-effect; placebo; ADHD; Type III errors; non-inert shams

Citation: Trullinger, M., Novian, A., Russel-Chapin, L., \& Pradhan, D. (2019). Perspectives on Type III statistical errors: Exaggerating the effects of placebo in neurofeedback. NeuroRegulation, 6(1), 38-41. http://dx.doi.org/10.15540/nr.6.1.38

*Address correspondence to: Mark Trullinger, 1205 York Rd, Suite

11, Lutherville, MD 21093, USA. Email: neurothrive@gmail.com

Copyright: (c) 2019. Trullinger et al. This is an Open Access article distributed under the terms of the Creative Commons Attribution License (CC-BY).

\section{Edited by:}

Nancy L. Wigton, PhD, Grand Canyon University, Phoenix, Arizona, USA

\section{Reviewed by:}

John Davis, PhD, McMaster University, Hamilton, Ontario, Canada Randall Lyle, PhD, Mount Mercy University, Cedar Rapids, lowa, USA
Reviews of electroencephalography neurofeedback (EEG-nf) have led to an often published, yet flawed, conclusion that EEG-nf research does not support efficacy for the treatment of attentiondeficit/hyperactivity disorder (ADHD) and that effects of EEG-nf arise primarily from placebo (Ghaziri \& Thibault, 2019; Thibault, Lifshitz, Birbaumer, \& Raz, 2015; Thibault, Lifshitz, \& Raz, 2016, 2017a, 2017b; Thibault \& Raz, 2017; Thibault, Veissière, Olson, \& Raz, 2018). This analytical approach is commonly based on a literature review, specifically examining randomized placebo-controlled trials of EEG-nf with a preference towards higher amounts of blinding. However, these literature reviews commit at least two Type III statistical errors, as we illustrate below. We therefore suggest that the conclusions of inefficacy of
EEG-nf in the treatment of ADHD and the placebo explanation are misguided and invalid.

The argument against the efficacy of EEG-nf is based on the assumption that sham EEG-nf is inert, thus rendering it an effective sham. Sham control study designs require an inert sham, which is a fake treatment that does not have a significant specific (i.e., non-placebo) effect on the condition being studied, such as the effect of a sugar pill in medication studies (Thornton, 2018). Most EEG-nf studies lack inert shams, and instead use another active treatment as a sham. An active sham, or non-inert sham, is when the treatment in the sham condition has a significant positive effect on ADHD that is comparable to other known treatments or beyond what could be due to placebo. This means that these studies are 
actually comparing two active conditions, both of which are found to have a positive treatment effect on ADHD symptoms. Since such study questions hypothesize a result based on comparing EEG-nf to an inert sham, which would have no effect beyond placebo, it often appears as if EEG-nf is not creating a significant change in comparison to the sham control. This study design only works if the sham control does not create a statistically significant effect itself. Moreover, if learning occurs on the target EEG variables within the sham group, which is the active ingredient of EEG-nf, then the sham is rendered ineffective because it is actually a valid form of EEGnf.

The recent reviews of EEG-nf claiming a lack of efficacy in the treatment of ADHD, positing placebo as the explanation for any positive effect, base their argument on studies that contain a non-inert EEG-nf sham condition (Ghaziri \& Thibault, 2019; Thibault et al., 2015; Thibault et al., 2016, 2017a, 2017b; Thibault \& Raz, 2017; Thibault et al., 2018). The EEG-nf shams used in many of these cited studies are not inert because they have a significant and positive effect on ADHD symptoms, with effect sizes comparable to known effective treatments for ADHD (Shönenberg et al., 2017; Van Doren et al., 2018). When a study, or group of studies, does not find separation between an active (non-inert) sham and true EEG-nf, it is an illusion called a false no-effect.

In instances of a false no-effect, no conclusions are to be drawn from these studies on efficacy or the presence of placebo because they are entirely subjective and absent of objectivity (Horn, Balk, \& Gold, 2011). This flaw is well understood in complementary and alternative medicine (Horn et al., 2011), and the issue of active shams are specifically identified as being present in studies of EEG-nf in the treatment of ADHD by both supporters and detractors of EEG-nf (Loo \& Makeig, 2012; Van Doren et al., 2018). Thibault and Raz (2018) even pointed out that the sham in all but two of the studies they cite have a positive effect on ADHD symptoms (Logemann, Lansbergen, Van Os, Böcker, \& Kenemans, 2010; Vollebregt, van Dongen-Boomsma, Buitelaar, \& Slaats-Willemse, 2014). The two studies excluded from that statement did not follow best practices in the actual EEG-nf condition, which likely explains the lack of difference between the sham and attempted EEGnf (Pigott, Cannon, \& Trullinger, 2018). This creates an easy-to-make Type III statistical error, or an error in which the data is collected and analyzed correctly but while it rejects the null hypothesis, it does not confirm the hypothesis that the researchers originally proposed (Tate, 2015).

Due to the misinterpretation of the false no-effect in these trials as an actual lack of effect, researchers often provide an ad hoc explanation, or a specific reason given to explain why a significant difference is or is not shown in the data. However, presenting ad hoc explanations is not advisable because they are not the specific, or only, difference between conditions being studied (Tate, 2015). There are possible reasons, other than placebo, that could contribute the lack of difference between EEG-nf and sham. Yet, the placebo is often presented definitively as the source of positive effects from EEG-nf in reviews of the literature (Ghaziri \& Thibault, 2019; Thibault et al., 2015; Thibault et al., 2016, 2017a, 2017b; Thibault \& Raz, 2017; Thibault et al., 2018). The continual repetition of this ad hoc placebo explanation in publications damages the overall quality and reliability of the published research, especially in the evaluation of efficacy, because it is a Type III statistical error and an unsubstantiated conclusion.

The sham EEG-nf designs of studies cited by several recent publications (Ghaziri \& Thibault, 2019; Thibault et al., 2015; Thibault et al., 2016, 2017a, 2017b; Thibault \& Raz, 2017; Thibault et al., 2018) use sham conditions that have not been validated as effective. One example is van Dongen-Boomsma, Vollebregt, Slaats-Willemse, and Buitelaar (2013), in which the sham condition trained a simulated signal of the EEG not coming from the participant with an $80 \%$ positive reinforcement rate that was adjusted to maintain that reward level identical to the reward ratio and adjustments in the actual EEG-nf condition. The participants receiving this sham treatment would be told that they are attempting to change their brainwaves through doing better at the assigned task, or similar instructions, which were identical to those given to the participants in the actual EEG-nf group.

The sham used by van Dongen-Boomsma et al. (2013) may actually achieve a significant amount of contingent reinforcement on the target EEG variable. Therefore, the sham may not actually represent a different treatment from what was administered in the actual EEG-nf condition (Thatcher \& Lubar, 2014). Moreover, the only way to know for sure would be to analyze whether or not learning occurred on the target EEG variable in the sham condition. Yet, the authors of this study did not do that. Neither did the recent reviews that cited these articles when claiming a lack of efficacy for EEG-nf in the treatment of ADHD 
and explanatory placebo effects (Ghaziri \& Thibault, 2019; Thibault et al., 2015; Thibault et al., 2016, 2017a, 2017b; Thibault \& Raz, 2017; Thibault et al., 2018).

Another example is Shönenberg et al. (2017), in which the sham condition received the sham during only $50 \%$ of the sessions. The EEG-nf treatment used in the actual EEG-nf group made up the last $50 \%$ of the sessions in the sham condition. Clearly, this sham condition cannot be assumed to be effective, or very different from the actual EEG-nf condition, because half of the treatments were identical to those administered to the actual EEG-nf group. Therefore, the risk of a false no-effect error is even higher in Shönenberg et al. (2017) because of the similarity between the sham and actual EEG-nf conditions. These are just two examples, but an analysis of all of the citations provided by these recent reviews of the literature (Ghaziri \& Thibault, 2019; Thibault et al., 2015; Thibault et al., 2016, 2017a, 2017b; Thibault \& Raz, 2017; Thibault et al., 2018) reveals that the sham conditions may have had some level of contingent reinforcement on the targeted EEG signal and did not provide evidence to prove otherwise.

Since the EEG-nf sham likely achieved some level of contingent reinforcement in the studies cited in recent reviews due to poor behavior modification designs (Ghaziri \& Thibault, 2019; Thibault et al., 2015; Thibault et al., 2016, 2017a, 2017b; Thibault \& Raz, 2017; Thibault et al., 2018;), these sham conditions are actually another form of EEG-nf with a theoretically less optimal behavioral modification paradigm. Therefore, they are not an effective sham and certainly cannot be assumed to be inert. In an even more recent publication, Ghaziri and Thibault (2019) state that that EEG-nf is not efficacious and the majority of the effect is placebo. They go on to state that EEG-nf needs to be compared to a specific sham condition, training a recorded signal that is not coming from the actual participants' real-time EEG, to prove EEG-nf is efficacious and not due to placebo. However, they do not cite any new research since Thibault et al. (2018) that has tested this sham to prove it is effectively not reinforcing the target EEG-nf variable. Nor do they provide any evidence that it is inert and does not significantly improve ADHD symptomology.

Just as those who support the efficacy of EEG-nf have to prove it works beyond placebo and generalized treatment effects, when researchers argue that placebo explains all of the effects of EEG- nf, they too must provide convincing proof that all other explanations for the effect are incorrect and cite research that shows placebo accounts for all of the positive clinical effect. As has been pointed out in this article, those claiming that placebo explains all of the effects of EEG-nf (Ghaziri \& Thibault, 2019; Thibault et al., 2015; Thibault et al., 2016, 2017a, 2017b; Thibault \& Raz, 2017; Thibault et al., 2018) have only succeeded in committing two distinct Type III statistical errors. There simply is not sufficient research to support the idea that placebo can make significant and sustainable changes that can explain the entirety of the effect of EEG-nf in the treatment of ADHD.

In fact, there is direct evidence that EEG-nf cannot be fully explained by placebo. A recent meta-analysis proves that the effects of appropriately administered EEG-nf last over time, even after the effects of placebo would wear off (Van Doren et al., 2018). Moreover, a second recent reanalysis of Cortese et al. (2016) statistically calculated results in a subgroup of the data that used the best practices in EEG-nf for their true EEG-nf condition. The studies that used the best practices in the EEG-nf field yielded significant evidence that EEG-nf effects in the treatment of ADHD cannot be fully explained by placebo in shortterm studies (Bussalb et al., 2019) despite the presence of a non-inert sham. Therefore, professing that placebo can explain all of the effect of EEG-nf in the treatment of ADHD is unsupported by the literature and exaggerates the effects of placebo interventions.

In the future, it is recommended that journal reviewers, editors, book publishers, and the general media should require authors to prove assumptions that the shams used in cited studies are both inert and effective. Additionally, the publishers should require proof that the EEG-nf condition being evaluated is a form of EEG-nf that is in alignment with the best practices of the field in all of the studies being cited. Finally, ad hoc explanatory explanations, such as placebo, are a Type III statistical error and should be excluded from the publication or appropriately and clearly identified as an ad hoc explanation and a Type III statistical error. Future research should assess the efficacy of EEG-nf as a treatment for ADHD within a more objective framework designed for dealing with non-inert shams, complex behavioral modification principles that need to be effectively administered, and a multifactorial effect of the treatment. The American Psychological Association has criteria for evaluating psychotherapy that meet these requirements (Chambless \& Hollon, 1998). 


\section{Author Disclosure}

Mark Trullinger receives grant funding as a primary investigator from BrainFutures, is a board member of the Foundation for Neurofeedback and Neuromodulation Research, and is the managing director of NeuroThrive, LLC.

Allen Novian is the owner of integrative Counseling \& Neurofeedback Solutions (iCNS), practices EEG-nf, and is an adjunct professor at St. Mary's University.

\section{Lori Russell-Chapin is a Professor at Bradley} University and also practices EEG-nf.

Deepti Pradhan is the chief operating officer of NeuroThrive, LLC.

This specific submission was funded by a grant from BrainFutures.

\section{References}

Bussalb, A., Congedo, M., Barthélemy, Q., Ojeda, D., Acquaviva, E., Delorme, R., \& Mayaud, L. (2019). Clinical and experimental factors influencing the efficacy of neurofeedback in ADHD: A meta-analysis. Frontiers in Psychiatry, 10, 35. http://dx.doi.org/10.3389/fpsyt.2019.00035

Chambless, D. L., \& Hollon, S. D. (1998). Defining empirically supported therapies. Journal of Consulting and Clinical Psychology, 66(1), 7-18. http://dx.doi.org/10.1037/0022006X.66.1.7

Cortese, S., Ferrin, M., Brandeis, D., Holtmann, M., Aggensteiner, P., Daley, D., ... Sonuga-Barke, E. J. S. (2016). Neurofeedback for attention-deficit/hyperactivity disorder: Meta-Analysis of clinical and neuropsychological outcomes from randomized controlled trials. Journal of the American Academy of Child \& Adolescent Psychiatry, 55(6), 444-455. http://dx.doi.org/10.1016/j.jaac.2016.03.007

Ghaziri, J., \& Thibault, R. T. (2019). Neurofeedback: An inside perspective. In A. Raz \& R. T. Thibault (Eds.), Casting Light on the Dark Side of Brain Imaging (pp. 113-116). Cambridge, MA: Academic Press. http://dx.doi.org/10.1016/B978-0-12-8161791.00019-0

Horn, B., Balk, J., \& Gold, J. I. (2011). Revisiting the sham: Is it all smoke and mirrors? Evidence-Based Complementary and Alternative Medicine, 2011, 842767. http://dx.doi.org/10.1093 lecam $/$ neq074

Logemann, H. N. A., Lansbergen, M. M., Van Os, T. W. D. P., Böcker, K. B. E., \& Kenemans, J. L. (2010). The effectiveness of EEG-feedback on attention, impulsivity and EEG: A sham feedback controlled study. Neuroscience Letters, 479(1), 4953. http://dx.doi.org/10.1016/j.neulet.2010.05.026

Loo, S. K., \& Makeig, S. (2012). Clinical utility of EEG in attentiondeficit/hyperactivity disorder: A research update. Neurotherapeutics, 9(3), 569-587. http://dx.doi.org/10.1007 /s13311-012-0131-z

Pigott, H. E., Cannon, R., \& Trullinger, M. (2018). The fallacy of sham-controlled neurofeedback trials: A reply to Thibault and colleagues (2018). Journal of Attention Disorders, 1087054718790802 . http://dx.doi.org/10.1177 /1087054718790802
Schönenberg, M., Wiedemann, E., Schneidt, A., Scheeff, J., Logemann, A., Keune, P. M., \& Hautzinger, M. (2017). Neurofeedback, sham neurofeedback, and cognitivebehavioural group therapy in adults with attention-deficit hyperactivity disorder: A triple-blind, randomised, controlled trial. The Lancet Psychiatry, 4(9), 673-684. http://dx.doi.org /10.1016/S2215-0366(17)30291-2

Tate, C. U. (2015). Type III and Type IV errors: Statistical decisionmaking considerations in addition to rejecting and retaining the null hypothesis. Retrieved from: http://web.stanford.edu/group/bps/cgibin/wordpress/wpcontent/uploads/2015/06/Tate.pd

Thatcher, R. W., \& Lubar, J. F. (Eds.). (2014). Z score neurofeedback: Clinical applications. San Diego, CA: Academic Press.

Thibault, R. T., Lifshitz, M., Birbaumer, N., \& Raz, A. (2015). Neurofeedback, self-regulation, and brain imaging: Clinical science and fad in the service of mental disorders. Psychotherapy and Psychosomatics, 84(4), 193207. http://dx.doi.org/10.1159/000371714

Thibault, R. T., Lifshitz, M., \& Raz, A. (2016). The self-regulating brain and neurofeedback: Experimental science and clinical promise. Cortex, 74, 247-261. http://dx.doi.org/10.1016 /j.cortex.2015.10.024

Thibault, R. T., Lifshitz, M., \& Raz, A. (2017a). Neurofeedback or neuroplacebo? Brain, 140(4), 862-864. http://dx.doi.org /10.1093/brain/awx033

Thibault, R. T., Lifshitz, M., \& Raz, A. (2017b). The climate of neurofeedback: Scientific rigour and the perils of ideology. Brain, 141(2), e11. http://dx.doi.org/10.1093/brain lawx330

Thibault, R. T., \& Raz, A. (2017). The psychology of neurofeedback: Clinical intervention even if applied placebo. American Psychologist, 72(7), 679-688. http://dx.doi.org/10.1037/amp0000118

Thibault, R. T., Veissière, S., Olson, J. A., \& Raz, A. (2018). Treating ADHD with suggestion: Neurofeedback and placebo therapeutics. Journal of Attention Disorders, 22(8), 707-711. http://dx.doi.org/10.1177/1087054718770012

Thornton, K. E. (2018). Perspectives on placebo: The psychology of neurofeedback. NeuroRegulation, 5(4), 137-149. http://dx.doi.org/10.15540/nr.5.4.137

van Dongen-Boomsma, M., Vollebregt, M. A., Slaats-Willemse, D., \& Buitelaar, J. K. (2013). A randomized placebo-controlled trial of electroencephalographic (EEG) neurofeedback in children with attention-deficit/hyperactivity disorder. The Journal of Clinical Psychiatry, 74(8), 821-827. http://dx.doi.org/10.4088/JCP.12m08321

Van Doren, J., Arns, M., Heinrich, H., Vollebregt, M. A., Strehl, U., \& Loo, S. K. (2018). Sustained effects of neurofeedback in ADHD: A systematic review and meta-analysis. European Child \& Adolescent Psychiatry, 28(3), 293-305. http://dx.doi.org/10.1007/s00787-018-1121-4

Vollebregt, M. A., van Dongen-Boomsma, M., Buitelaar, J. K., \& Slaats-Willemse, D. (2014). Does EEG-neurofeedback improve neurocognitive functioning in children with attentiondeficit/hyperactivity disorder? A systematic review and a double-blind placebo-controlled study. The Journal of Child Psychology and Psychiatry, and Allied Disciplines, 55(5), 460472. http://dx.doi.org/10.1111/jcpp.12143

Received: March 3, 2019

Accepted: March 21, 2019

Published: March 25, 2019 